\title{
CDISC SDTM Cardiovascular Findings About Results Terminology
}

National Cancer Institute

\section{Source}

National Cancer Institute. CDISC SDTM Cardiovascular Findings About Results

Terminology. NCl Thesaurus. Code C119016.

Terminology associated with the cardiovascular findings about results codelist of the Clinical Data Interchange Standards Consortium (CDISC) Study Data Tabulation Model (SDTM). 